\title{
Almost All Finsler Metrics have Infinite Dimensional Holonomy Group
}

\author{
B. Hubicska ${ }^{1}$. V. S. Matveev ${ }^{2}$ (D) Z. Muzsnay ${ }^{1}$ (D)
}

Received: 21 July 2020 / Accepted: 4 September 2020 / Published online: 24 September 2020

(C) The Author(s) 2020

\begin{abstract}
We show that the set of Finsler metrics on a manifold contains an open everywhere dense subset of Finsler metrics with infinite-dimensional holonomy groups.
\end{abstract}

Keywords Finsler geometry · Algebras of vector fields · Holonomy · Curvature

Mathematics Subject Classification 53C29 - 53B40 - 22E65 - 17B66.

\section{Introduction}

Finsler metrics appeared already in the inaugural lecture of Riemann in 1854 [19], under the name generalized metric. At the beginning of the XXth century, the intensive study of Finsler metrics was motivated by the optimal transport theory. A group of mathematicians lead by Cartheodory aimed to adapt mathematical tools which were effective in Riemannian geometry (such as affine connections, Jacobi vector fields, sectional curvature) for a more general situation. Finsler was a student of Cartheodory and his dissertation [5] is one of the important steps on this way.

Riemannian geometry is one of the main sources of challenging problems in Finsler geometry: many Riemannian results are not valid in the Finslerian setup and one asks under what additional assumptions they are correct.

Our paper studies the holonomy groups of Finsler manifolds. We give precise definitions later; at the present point let us recall that the Berwald connection (introduced

\footnotetext{
Z. Muzsnay

muzsnay@science.unideb.hu

B. Hubicska

hubicska.balazs@science.unideb.hu

V. S. Matveev

vladimir.matveev@uni-jena.de

1 University of Debrecen, Institute of Mathematics, Pf. 400, Debrecen 4002, Hungary

2 Friedrich-Schiller-Universität, Institut für Mathematik, 07737 Jena, Germany
} 
by Berwald in 1926 [2]) can be viewed as an Ehresmann connection on the unit tangent bundle $\mathcal{I} M$. Its holonomy group (at $x \in M$ ) is the subgroup of the group $\operatorname{Diff}\left(\mathcal{I}_{x}\right)$ generated by the parallel transports along the loops starting and ending at $x$.

For Riemannian metrics, the Berwald connection specifies to the Levi-Civita connection. Study of Riemannian holonomy groups is a prominent topic in Riemannian geometry and mathematical physics. It is known (see e.g. Borel and Lichnerowicz [3]) that the holonomy group is a subgroup of the orthogonal group; in particular it is always finite-dimensional. Moreover, all possible holonomy groups are described and classified due in particular to breakthrough results of Berger and Simons [1,20].

In the Finslerian case, the situation is very different and not much is known. By [21] (see also [12,13,22]) the so-called Berwald manifolds have finite-dimensional holonomy group. Also the so-called Landsberg manifolds have finite-dimensional holonomy group [10], but it is not jet known whether nonberwaldian Landsberg manifolds exist [11]. We are not aware of other examples of Finsler metrics with finite-dimensional holonomy group, it is an interesting problem to find such.

From the other side, there are also not many explicit examples of Finsler manifolds with infinite-dimensional holonomy group [7,16], and all these examples have constant curvature. A natural and fundamental question in this context is whether for a generic Finsler manifold the holonomy group is infinite-dimensional. Its simplest version was explicitly asked by Chern et al in [4, p. 85].

In our paper, we prove that for a generic Finsler manifold the holonomy group is infinite-dimensional:

Theorem 1.1 In the set $\mathcal{F}$ of $C^{\infty}$-smooth Finsler metrics on a manifold $M$ of dimension $n \geq 2$, there exists a subset $\widetilde{\mathcal{F}}$ of Finsler metrics with infinite dimensional holonomy group, which is open and everywhere dense in any $\mathcal{C}^{m}$-topology, $m \geq 8$.

What we essentially prove is that one can $C^{\infty}$ small perturb any Finsler metric $F$ at a neighbourhood of any point $x \in M$ such that for every open nonempty subset $U \subseteq M$ containing $x$, the perturbed Finsler structure $\left(U, F_{t}\right)$ has infinite-dimensional holonomy group. Moreover, the metric $F_{t}$ has an open subset in the space of all $C^{\infty}$ Finsler metrics equipped with $C^{m \geq 8}$-topology such that every Finsler metric $F^{\prime \prime}$ in this set also has infinite-dimensional holonomy group. Theorem 1.1 is true mircrolocally and on the level of germs (see Remark 5.1). The perturbation is given by a formula. We show that for almost every $t \in[0,1]$, the perturbation $F_{t}$ on its indicatrix $\mathcal{I}_{x}^{t}$ has the full infinity-jet at every point $y \in \mathcal{I}_{x}^{t}$. Based on this, we conjecture that in the generic case, the holonomy group of a Finsler manifold coincides with the full diffeomorphism group of the indicatrix.

Our results imply that, in contrast to the Riemannian case, the closure of the holonomy group is not a compact group for most Finlser metric. Similar results for the linear holonomy group (defined via the linear parallel transport) were recently obtained in [8].

The proof is organised as follows. We first show that the standard Funk metric $F_{F u n k}$ has 'sufficiently large' holonomy algebra. For dimension 2, this was known $[7,16]$, we generalise these results to all dimensions. Next, we employ the trick from $[14, \S 3.1]$ and show that with the help of $F_{F u n k}$ one can perturb an arbitrary Finsler metric such the result also has 'sufficiently large' holonomy algebra. Then, we show 
that if the holonomy algebra is 'sufficiently large' then it is infinite-dimensional. This step is in fact a general statement about algebras of smooth vector fields and possibly can be applied elsewhere. Therefore, let us formulate it as Theorem 1.2 below, this will also explain what we understand by 'sufficiently large'.

An algebra $\mathfrak{g}$ of vector fields on $U \subseteq \mathbb{R}^{n}$ is called 3-jet generating at $x \in U$, if the set of 3rd jets of these vector fields at the point $x$ coincides with the space of all 3rd jets of vector fields at $x$ (see Definition 3.3). In other words, every vector field can be approximated at $x$ with order three by a vector field from the algebra. For example, if the algebra is locally transitive at $x$, i.e., if the elements of the algebra at $x$ span the whole $T_{x} U$, then it is 0 -jet generating.

Theorem 1.2 Let $\mathfrak{g}$ be a Lie algebra of vector fields on a manifold $U$. If there exists a point where it is 3-jet generating, then $\mathfrak{g}$ is infinite-dimensional.

If dimension $U$ is 1 , the result is known and is due to Sophus Lie, see e.g. [17, Theorem 2.70]. As examples show (see e.g. the tables at the back of [17] where vector field algebras of arbitrary finite dimension are listed), the 3 -jet generating property is important.

\section{Preliminaries}

Let $M$ be an $n$-dimensional manifold, $T M$ its tangent manifold, $\pi: T M \rightarrow M$ the canonical projection. Local coordinates $\left(x_{i}\right)$ on $M$ induce local coordinates $\left(x_{i}, y_{i}\right)$ on $T M$. The $k$ th order jet of a function $f \in C^{\infty}(M)$ (resp. smooth vector field $V \in \mathfrak{X}(M)$ ) at $x \in M$ will be denoted by $j_{x}^{k}(f)$ (resp. $j_{x}^{k}(V)$ ). In local coordinates, the $k$ th order jet can be viewed as the collection of all derivatives of the function or of the vector field up to the order $k$. For example, the 1 st order jet of a function at a point $x$ can be viewed as $n+1$ numbers $\left(f, \frac{\partial f}{\partial x_{1}}, \ldots, \frac{\partial f}{\partial x_{n}}\right)$, and of a vector field $V$ as $n(n+1)$ numbers $\left(V_{1}, \ldots, V_{n}, \frac{\partial V_{1}}{\partial x_{1}}, \ldots, \frac{\partial V_{n}}{\partial x_{n}}\right)$.

\subsection{Finsler Manifolds, Connection}

The function $F: T M \rightarrow \mathbb{R}_{+}$is called a Finsler metric, if it is a positively 1homogeneous continuous function, $C^{\infty}$-smooth on $\widehat{T} M=T M \backslash\{0\}$ and

$$
g_{i j}=\frac{\partial^{2} \mathcal{E}}{\partial y_{i} \partial y_{j}}
$$

is positive definite at every $y \in \widehat{T}_{x} M$, where $\mathcal{E}:=\frac{1}{2} F^{2}$ denotes the energy function of $F$. A pair $(M, F)$ is called a Finsler manifold. The hypersurface of $T_{x} M$ defined by

$$
\mathcal{I}_{x}=\left\{y \in T_{x} M: F_{x}(y)=1\right\}
$$

is called the indicatrix at $x \in M$. The geodesics of a Finsler manifold $(M, F)$ are given by the solutions of the following system of second-order ordinary differential equations 


$$
\ddot{x}^{i}+2 G^{i}(x, \dot{x})=0,
$$

where the geodesic coefficients $G^{i}=G^{i}(x, y)$ are determined by the formula

$$
G^{i}=\frac{1}{4} g^{i l}\left(2 \frac{\partial g_{j l}}{\partial x_{k}}-\frac{\partial g_{j k}}{\partial x_{l}}\right) y_{j} y_{k}, \quad i=1, \ldots, n .
$$

The (Berwald) parallel translation on a Finsler manifold can be introduced by considering the Ehresmann connection: the horizontal distribution is determined by the image of the horizontal lift $T_{x} M \rightarrow T_{(x, y)} T M$ defined in a local basis as

$$
\delta_{i}:=\left(\frac{\partial}{\partial x_{i}}\right)^{h}=\frac{\partial}{\partial x_{i}}-G_{i}^{k} \frac{\partial}{\partial y_{k}}
$$

where $y \in \widehat{T}_{x} M$ and $G_{j}^{i}=\frac{\partial G^{i}}{\partial y_{j}}$. We have the decomposition

$$
T T M=\mathcal{H} \oplus \mathcal{V}
$$

where $\mathcal{V}=\operatorname{ker} \pi_{*}$ is the vertical distribution. The corresponding projectors are denoted by $h$ and $v$. The horizontal Berwald covariant derivative of a vertical vector field $\xi$ with respect to a vector field $X \in \mathfrak{X}(M)$ is defined by

$$
\nabla_{X} \xi=\left[X^{h}, \xi\right]
$$

In local coordinates, if $\xi=\xi^{i}(x, y) \frac{\partial}{\partial y_{i}}$ and $X(x)=X^{i} \frac{\partial}{\partial x_{i}}$, then

$$
\nabla_{X} \xi=\left(\frac{\partial \xi^{i}}{\partial x_{j}}-G_{j}^{k} \frac{\partial \xi^{i}}{\partial y_{k}}+\frac{\partial G_{j}^{i}}{\partial y_{k}} \xi^{k}\right) X_{j} \frac{\partial}{\partial y_{i}} .
$$

\subsection{Parallel Translation and Curvature}

Parallel vector fields along a curve $c$ are characterized by the property that their covariant derivative vanishes. Parallel translation can be obtained through the following geometric construction: the horizontal lift of a curve $c:[0,1] \rightarrow M$ with initial condition $X_{0} \in T_{c(0)} M$ is a curve $c^{h}:[0,1] \rightarrow T M$ such that $\pi \circ c^{h}=c, \frac{d c^{h}}{d t}=\left(\frac{d c}{d t}\right)^{h}$ and $c^{h}(0)=X_{0}$. Then the parallel translation of $X_{0}$ along the curve $c$ from $c(0)$ to $c(1)$ is

$$
\mathcal{P}_{c}\left(X_{0}\right)=c^{h}(1)
$$

The horizontal distribution $\mathcal{H}$ is, in general, non-integrable. The obstruction to its integrability is given by the curvature tensor $R=\frac{1}{2}[h, h]$ which is the Nijenhuis torsion of the horizontal projector $h$ associated to the subspace $\mathcal{H}$. The curvature tensor field is defined by

$$
R=R_{j k}^{i}(x, y) \mathrm{d} x_{j} \otimes \mathrm{d} x_{k} \otimes \frac{\partial}{\partial y_{i}}
$$


where

$$
R_{j k}^{i}=\frac{\partial G_{j}^{i}}{\partial x_{k}}-\frac{\partial G_{k}^{i}}{\partial x_{j}}+G_{j}^{m} G_{k m}^{i}-G_{k}^{m} G_{j m}^{i}
$$

in a local coordinate system with $G_{j k}^{i}=\frac{\partial G_{j}^{i}}{\partial y_{k}}$.

Remark 2.1 From formula (3) we get that the geodesic coefficients $G^{i}(x, y)$ can be calculated in terms of the 3rd order jet of the Finsler function $F$ at $(x, y)$. Therefore, the coefficients $R_{j k}^{i}(x, y)$ of the curvature tensor and the curvature vector fields

$$
\mathcal{R}_{i j}:=R\left(\delta_{i}, \delta_{j}\right), \quad i, j=1, \ldots, n,
$$

can be expressed algebraically by the 5 th order jet of $F$. More generally, the value of $k$ th order covariant derivatives and $k$ th successive Lie brackets of curvature vector fields can be expressed algebraically by the $(k+5)$ th order jet of $F$.

\subsection{The Holonomy Group, the Holonomy Algebra and Its Subalgebras}

The holonomy group $\operatorname{Hol}_{x}(M, F)$ of a Finsler manifold $(M, F)$ at a point $x \in M$ is the group generated by parallel translations along piece-wise differentiable closed curves starting and ending at $x$. Since the parallel translation (5) is 1-homogeneous and preserves the norm, one can consider it as a map

$$
\mathcal{P}_{c}: \mathcal{I}_{x} \rightarrow \mathcal{I}_{x}
$$

therefore, the holonomy group can be seen as a subgroup of the diffeomorphism group of the indicatrix:

$$
\mathcal{H}_{\text {ol }}(M, F) \subset \mathcal{D i f f}\left(\mathcal{I}_{x}\right)
$$

and its tangent space at the identity, is called the holonomy algebra:

$$
\mathfrak{h o l}_{x}(M, F) \subset \mathfrak{X}\left(\mathcal{I}_{x}\right) .
$$

We are listing below the most important properties of the holonomy algebra (see [7]):

Property $2.2(1) \mathfrak{h o l}_{x}(M, F)$ is a Lie subalgebra of $\mathfrak{X}\left(\mathcal{I}_{x}\right)$,

(2) the exponential image of $\mathfrak{h o l}_{x}(M, F)$ is in the topological closure of $\mathcal{H}_{x}(M, F)$.

The infinitesimal holonomy algebra $\mathfrak{h o l}_{x}^{*}(M, F)$ is generated by curvature vector fields and their horizontal Berwald covariant derivatives, that is:

$$
\mathfrak{h o l}_{x}^{*}(M, F):=\left\langle\nabla_{Z_{1}} \ldots \nabla_{Z_{k}} R\left(X^{h}, Y^{h}\right) \mid X, Y, Z_{1}, \ldots, Z_{k} \in \mathfrak{X}(M)\right\rangle_{\text {Lie }} .
$$

The infinitesimal holonomy algebra $\mathfrak{h o l}_{x}^{*}(M, F)$ is a Lie subalgebra of $\mathfrak{h o l}_{x}(M, F)$. 
Remark 2.3 The infinitesimal holonomy algebra is local in nature, that is for any open neighbourhood $U$ of $x \in M$ we get $\mathfrak{h o l}_{x}^{*}(M, F)=\mathfrak{h o l}_{x}^{*}\left(U,\left.F\right|_{\pi^{-1}(U)}\right)$. For that reason, we will simplify the notation

$$
\mathfrak{h o r} \mathfrak{l}_{x}^{*}(F):=\mathfrak{h o l}_{x}^{*}(M, F)
$$

by omitting the neighborhood of the point where the infinitesimal holonomy algebra is determined. Indeed, the curvature vector fields, their horizontal Berwald covariant derivatives and their Lie brackets can be computed on an arbitrarily small neighbourhood of $x$, therefore, their value at $x$ can be determined locally.

We have the inclusions of Lie algebras:

$$
\mathfrak{h o l}_{x}^{*}(F) \subset \mathfrak{h o l}_{x}(M, F) \subset \mathfrak{X}\left(\mathcal{I}_{x}\right)
$$

therefore, at the level of groups, we get

$$
\exp \left(\mathfrak{h o l} \mathfrak{l}_{x}^{*}(F)\right) \subset \exp \left(\mathfrak{h o l}_{x}(M, F)\right) \subset \mathcal{H} l_{x}^{c}(M, F) \subset \mathcal{D} \text { iff }\left(\mathcal{I}_{x}\right)
$$

where $\mathcal{H}_{c}^{c}(M)$ denotes the topological closure of the holonomy group with respect to the $C^{\infty}$-topology of $\mathcal{D}$ iff $\left(\mathcal{I}_{x}\right)$. We call a Lie algebra infinite dimensional if it contains infinitely many $\mathbb{R}$-linearly independent elements. Clearly, using the tangent property of the holonomy algebra, if $\mathfrak{h o l}_{x}(M, F)$ is infinite dimensional, then the holonomy group cannot be a finite dimensional Lie group. This observation motivates the following

Definition 2.4 The holonomy group of a Finsler manifold $(M, F)$ is called infinite dimensional if its holonomy algebra is infinite dimensional.

We refer to [7] for a discussion of the tangent Lie algebras of diffeomorphism groups and of the relation between the holonomy group and the holonomy algebra.

\section{On the Holonomy of the Standard Funk Metric}

A Funk metric can be described as follows. Let $\Omega$ be a bounded convex domain in $\mathbb{R}^{n}$ and denote its boundary by $\partial \Omega$. We can define a Finsler norm function $F_{\Omega}(x, y)$ in the interior of $\Omega$ for any vector $y \in T_{x} \Omega$ by the formulas

$$
F_{\Omega}(x, y)>0, \quad x+\frac{y}{F_{\Omega}(x, y)}=z,
$$

where $z \in \partial \Omega$. This norm function is called the Funk norm function induced by $\Omega$. The Funk norm induced by the origo centered unit ball $B^{n} \subset \mathbb{R}^{n}$ will be called the standard Funk norm and will be denoted by $F_{\mathbb{B}^{n}}$. We denote by $o=(0, \ldots, 0)$ the origin in $\mathbb{R}^{n}$. 
Remark 3.1 The holonomy of $\left(\mathbb{B}^{2}, F_{\mathbb{B}^{2}}\right)$ was investigated in [16, Chapter 5]. It was proved that the infinitesimal holonomy algebra $\mathfrak{h o l}_{o}^{*}\left(F_{\mathbb{B}^{2}}\right)$ contains the Fourier algebra $\mathrm{F}\left(\mathbb{S}^{1}\right)$ whose elements are vector fields $f \frac{d}{d t}$ such that $f(t)$ has finite Fourier series. One has

$$
\mathrm{F}\left(\mathbb{S}^{1}\right) \subset \mathfrak{h o l} \mathfrak{l}_{o}^{*}\left(F_{\mathbb{B}^{2}}\right) \subset \mathfrak{X}\left(\mathbb{S}^{1}\right) .
$$

Since $F\left(\mathbb{S}^{1}\right)$ is dense in $\mathfrak{X}\left(\mathbb{S}^{1}\right)$, we get the same from $(14)$ for $\mathfrak{h o l}_{o}^{*}\left(F_{\mathbb{B}^{2}}\right)$. Using the exponential map, one can obtain from (13) that the closure of the holonomy group of the Finsler surface $\left(\mathbb{B}^{2}, F_{\mathbb{B}^{2}}\right)$ is $\mathcal{D} i f f_{+}\left(\mathbb{S}^{1}\right)$, the group of orientation preserving diffeomorphisms of the circle [16, Theorem 5.2].

Proposition 3.2 The infinitesimal holonomy algebra of the standard Funk metric $F_{\mathbb{B}^{n}}$ at $o \in \mathbb{R}^{n}$ is infinite dimensional.

Proof For $n=2$ the proof follows directly from Remark 3.1 since (14) shows that $\mathfrak{h o l} \mathfrak{l}_{o}^{*}\left(F_{\mathbb{B}^{2}}\right)$ contains the infinite dimensional Lie algebra $\mathrm{F}\left(\mathbb{S}^{1}\right)$.

Let us consider the $n>2$ case. For each tangent 2-plane $\mathcal{K} \subset T_{o} \mathbb{B}^{n}$ the restriction of $F_{\mathbb{B}^{n}}$ to $\mathbb{B}^{2}:=\mathbb{B}^{n} \cap \mathcal{K}$ is the standard Funk metric on $\mathbb{B}^{2}$. One can suppose that $\mathcal{K}$ is the 2-plane generated by $\frac{\partial}{\partial x_{1}}$ and $\frac{\partial}{\partial x_{2}}$. Then, using the totally geodesic property, the curvature vector field and its successive covariant derivatives with respect to the directions $\frac{\partial}{\partial x_{1}}$ and $\frac{\partial}{\partial x_{2}}$ on $\mathbb{B}^{2}$ at $o$ can be inherited as restriction of the corresponding vector fields of $\mathbb{B}^{n}$. Consequently, the elements of the Fourier algebra can be obtained as the restriction of elements of the infinitesimal holonomy algebra $\mathfrak{h o l}^{*}\left(F_{\mathbb{B}^{n}}\right)$ and we have

$$
\mathrm{F}\left(\mathbb{S}^{1}\right) \subset \mathfrak{h o l}_{o}^{*}\left(F_{\mathbb{B}^{2}}\right) \simeq \mathfrak{h o l}_{o}^{*}\left(\left.F_{\mathbb{B}^{n}}\right|_{\mathbb{B}^{n} \cap \mathcal{K}}\right) \subset \mathfrak{h o l}_{o}^{*}\left(F_{\mathbb{B}^{n}}\right)
$$

It follows that $\mathfrak{h o l}_{o}^{*}\left(F_{\mathbb{B}^{n}}\right)$ contains infinitely many $\mathbb{R}$-independent vector fields which can be expressed by the curvature vector fields and their covariant derivatives.

Definition 3.3 A set $\mathcal{V} \subset \mathfrak{X}(M)$ of vector fields on a manifold $M$ is called

(1) $k$-jet generating at $x \in M$ if the natural map $j_{x}^{k}: \mathcal{V} \rightarrow J_{x}^{k}(\mathfrak{X}(M))$ is surjective,

(2) jet generating on $M$ if at any $x \in M$ and for any $k \geq 0$ it is $k$-jet generating.

We have the following

Proposition 3.4 The infinitesimal holonomy algebra $\mathfrak{h o l}_{o}^{*}\left(F_{\mathbb{B}^{n}}\right)$ of the standard Funk metric at the point $o \in \mathbb{B}^{n}$ has the jet generating property on the indicatrix $\mathcal{I}_{o}$.

Proof According to Definition 3.3, we have to show that for any $y \in \mathcal{I}_{o}$ and $k \in \mathbb{N}$ the jet-projection $\mathfrak{h o r}_{o}^{*}\left(F_{\mathbb{B}^{n}}\right) \rightarrow J_{y}^{k}\left(\mathfrak{X}\left(\mathcal{I}_{o}\right)\right)$ is onto.

In the case $n=2$, we get from Remark 3.1 that $\mathfrak{h o l}_{o}^{*}\left(F_{\mathbb{B}^{2}}\right)$ is dense in $\mathfrak{X}\left(\mathcal{I}_{o}\right)$. It follows that the restriction of the $k^{\text {th }}$ order jet projection on the infinitesimal holonomy algebra

$$
j_{y}^{k}: \mathfrak{h o} \mathfrak{l}_{o}^{*}\left(F_{\mathbb{B}^{2}}\right) \longrightarrow J_{y}^{k}\left(\mathfrak{X}\left(\mathcal{I}_{o}\right)\right),
$$

is onto. In other words, any given $k$ th order jet in $J_{y}^{k}\left(\mathfrak{X}\left(\mathcal{I}_{o}\right)\right)$ can be realized as the $k$-jet of an element of the infinitesimal holonomy algebra. Clearly we have the jet generating property. 
Let us consider the $n>2$ case. If $y \in \mathcal{I}_{o}$ and $v \in T_{y}\left(\mathcal{I}_{o}\right)$, let $\mathcal{K}_{y, v}$ be the 2-plane determined by these vectors. Using the argument of the proof of Proposition 3.2 and (15) we get that

$$
j_{y}^{k}: \mathfrak{h o r}_{o}^{*}\left(\left.F_{\mathbb{B}^{n}}\right|_{\mathcal{I}_{o} \cap \mathcal{K}_{y, v}}\right) \longrightarrow J_{y}^{k}\left(\mathfrak{X}\left(\mathcal{I}_{o} \cap \mathcal{K}_{y, v}\right)\right)
$$

is onto. It follows that for $y \in \mathcal{I}_{o}$ and $v \in T_{y}\left(\mathcal{I}_{o}\right)$, any $k^{\text {th }}$ order $v$-directional derivative can be realised by elements of the holonomy algebra. Using the local coordinate system $y_{1}, \ldots, y_{n-1}$ on the $n-1$-dimensional indicatrix $\mathcal{I}_{o}$ we get that for any given $\left(z, z_{1}, \ldots, z_{k}\right) \in\left(\mathbb{R}^{(n-1)}\right)^{(k+1)}$ there exist $\xi \in \mathfrak{h o}{ }_{o}^{*}\left(F_{\mathbb{B}^{n}}\right)$ such that

$$
\left.\xi\right|_{y}=z,\left.\quad\left(\mathcal{D}_{v} \xi\right)\right|_{y}=z_{1},\left.\quad \ldots \quad\left(\mathcal{D}_{v}^{(k)} \xi\right)\right|_{y}=z_{k},
$$

where we consider a locally constant extension of $v$ when the higher order derivatives are computed. For the completion of the proof, however, we must be able to generate all $k$-th jet at $y$, that is the terms corresponding to mixed partial derivatives as well. This is possible, by using higher order derivatives corresponding to several directions. Indeed, one can use the polarization technique to show that the $k^{\text {th }}$ order mixed partial derivatives are determined by the $k^{\text {th }}$ order directional derivatives, a similar way as the quadratic form determines the corresponding symmetric bilinear form, or more generally, as the homogeneous from of degree $k$ determines the corresponding symmetric multilinear $k$-from. Indeed, considering any $v_{1}, \ldots v_{k} \in T_{y}\left(\mathcal{I}_{o}\right)$ and their constant extension in a neighbourhood of $y$, we get

$$
\mathcal{D}_{v_{1}}\left(\mathcal{D}_{v_{2}} \cdots\left(\mathcal{D}_{v_{k}} \xi\right)\right)=\frac{1}{k !} \sum_{s=1}^{k} \sum_{1 \leq j_{1}<\cdots<j_{s} \leq k}(-1)^{k-s} \mathcal{D}_{v_{j_{1}}+\cdots+v_{j_{s}}}^{(k)} \xi .
$$

It follows that any mixed derivative can be realized for appropriately chosen (higher order) directional derivatives, therefore the $k$-jet generating property is satisfied. The argument works for any $y \in \mathcal{I}_{o}$ and $k \in \mathbb{N}$, therefore, the jet generating property holds.

Remark 3.5 [The jet generating property of curvature vector fields and their derivatives] One can easily show that in the 2-dimensional case, at any point $y \in \mathcal{I}_{o}$, the set of the curvature vector field and its derivatives up to order $k$ contains $k+1$ linearly independent $k$-jet, therefore, this set has the $k$-jet generating property. In the higher dimensional cases, from the argument of Proposition 3.4 using 2-dimensional planes, one can obtain that for any point $y \in \mathcal{I}_{o}$ and any direction $v \in T_{y}\left(\mathcal{I}_{o}\right)$, the curvature vector fields and their derivatives up to order $k$ can be used to express the directional derivatives (17). From formula (18) one obtains that any $k^{\text {th }}$ order derivative can be obtained by the derivatives of the curvature vector fields up to order $k$, that is the set

$$
\left\{\mathcal{R}_{i j}, \nabla_{p_{1}} \mathcal{R}_{i j}, \ldots, \nabla_{p_{1} \ldots p_{k}} \mathcal{R}_{i j} \mid 1 \leq i, j, p_{1} \ldots p_{k} \leq n\right\} \subset \mathfrak{X}\left(\mathcal{I}_{o}\right)
$$

has the $k$-jet generating property. 


\section{The Funk-Perturbed Finsler Metrics}

In this section, we investigate the holonomy group of a Finsler metric perturbed with the standard Funk metric. We present some technical properties of the Funk deformation which are essential in the proof of Theorem 1.1.

Let $(M, F)$ be a Finsler manifold and $x_{0} \in M$ be a fixed point. We can chose an $x_{0}$-centered coordinate system $(U, x)$ such that $x(U) \subset \mathbb{B}^{n}$. The associated coordinate system on $T M$ will be denoted by $\left(\pi^{-1}(U), \chi=(x, y)\right)$. We also consider a bump function $\psi: M \rightarrow \mathbb{R}$, such that $\operatorname{supp}(\psi) \subset U$ and $\left.\psi\right|_{\tilde{U}}=1$ for some open neighbourhood $\tilde{U} \subset U$ of $x_{0}$. We denote by $\bar{\psi}:=\psi \circ \pi$ the pull-back of $\psi$ by the projection $\pi$.

Using the standard Funk norm function $F_{\mathbb{B}^{n}}$, we introduce the Finsler norm $\bar{F}$ : $T M \rightarrow \mathbb{R}$ by the formula

$$
\bar{F}^{2}=\psi \cdot\left(F_{\mathbb{B}^{n}} \circ \chi\right)^{2}+(1-\psi) \cdot F^{2} .
$$

We remark that $\bar{F}$ is the pull-back of the standard Funk norm function on $\pi^{-1}(\tilde{U})$.

Using (20) we define a smooth perturbation of the Finsler function $F$ as a 1parameter family of functions $F_{t}$, where

$$
F_{t}^{2}=(1-t) F^{2}+t \bar{F}^{2}, \quad t \in[0,1] .
$$

Then, $F_{t}$ is a 1-parameter family of Finsler metrics. Indeed, $F$ and $\bar{F}$ are positively 1-homogeneous continuous function, smooth on $\widehat{T} M$, therefore, $F_{t}$ verifies these properties. Moreover, taking the squares in (4) ensures that the bilinear form

$$
g_{i j}^{t}=(1-t) g_{i j}+t \bar{g}_{i j}, \quad t \in[0,1]
$$

of $F_{t}$ is positive definite as well.

Proposition 4.1 Any element of the infinitesimal holonomy algebra $\mathfrak{h o l}_{x_{0}}^{*}\left(F_{t}\right)$ can be expressed as an algebraic fraction of polynomials in $t$ whose coefficients are determined by $j_{x_{0}}^{k} F$ and $j_{x_{0}}^{k} \bar{F}$ for some $k \in \mathbb{N}$.

Proof The geodesic coefficients $G_{t}^{i}, i=1, \ldots, n$ of $F_{t}$ can be calculated in terms of $j_{x_{0}}^{3}\left(F_{t}\right)$, therefore, in terms of $t, j_{x_{0}}^{3}(F)$, and $j_{x_{0}}^{3}(\bar{F})$. More precisely, their expressions are algebraic fractions of polynomials in $t$ whose coefficients are determined by the third-order jets of $F$ and $\bar{F}$. Similarly, the curvature vector fields of $F_{t}$ can be expressed as algebraic fractions of polynomials in $t$ whose coefficients are determined by $j_{x_{0}}^{5}(F)$ and $j_{x_{0}}^{5}(\bar{F})$. More generally, using Remark 2.1, the value of $k$ th order covariant derivatives and $k$ th successive Lie brackets of curvature vector fields of $F_{t}$ can be expressed as algebraic fractions of polynomials in $t$ whose coefficients are determined by $j_{x_{0}}^{k+5}(F)$ and $j_{x_{0}}^{k+5}(\bar{F})$.

Proposition 4.2 For any $y_{0} \in \mathcal{I}_{o}$ the set of parameters $t \in[0,1]$, where the 3-jet generating property of the infinitesimal holonomy algebra $\mathfrak{h o l}_{x_{0}}^{*}\left(F_{t}\right) \subset \mathfrak{X}\left(\mathcal{I}_{x_{0}}^{t}\right)$ of the Funk perturbation (4) is not satisfied, is finite. 
Proof Let us suppose that $y_{0} \in \mathcal{I}_{x_{0}}^{t} \subset T_{x_{0}} M$ for every $t \in[0,1]$. If not, then we can consider $\widetilde{F}_{t}(x, y):=F_{t}(x, y) / F_{t}\left(x_{0}, y_{0}\right)$ which is just a rescaling with a constant for any given $t$, therefore it doesn't affect the jet generating property on the indicatrix.

From Proposition 3.4 we know that the Funk metric has the jet generating property, therefore for $t=1$, there are vector fields

$$
\left\{W_{1}, \ldots, W_{l}\right\} \in \mathfrak{h o l}_{x_{0}}^{*}\left(F_{t=1}\right)
$$

$l:=\operatorname{dim}\left(J_{y_{0}}^{3}\left(\mathfrak{X}\left(\mathcal{I}_{x_{0}}^{t=1}\right)\right)\right)$ in the infinitesimal holonomy algebra, such that any thirdorder jet at $y_{0}$ can be realized with their combination. Those vector fields are linear combination of curvature vector fields, their derivatives and their Lie brackets. Let us consider them for any $F_{t}, t \in[0,1]$. We get a set in the infinitesimal holonomy algebra of $F_{t}$ at $x_{0}$ :

$$
\left\{W_{1}(t), \ldots, W_{l}(t)\right\} \in \mathfrak{h o l}_{x_{0}}^{*}\left(F_{t}\right) .
$$

Using Proposition 4.1, these vector fields are algebraic fractions of polynomials in $t$ whose coefficients are determined by $j_{x_{0}}^{k}(F)$ and $j_{x_{0}}^{k}(\bar{F})$ for some $k \in \mathbb{N}$. It follows that the determinant of the $l \times l$ matrix composed by the $3^{\text {rd }}$ order jet coordinates of (23) at $y_{0}$ :

$$
\mathcal{P}_{t}:=\operatorname{det}\left(\begin{array}{c}
j_{y_{0}}^{3}\left(W_{1}(t)\right) \\
\vdots \\
j_{y_{0}}^{3}\left(W_{l}(t)\right)
\end{array}\right),
$$

is an algebraic fraction of polynomials in $t$ whose coefficients are determined by $j_{x_{0}}^{3}(F)$ and $j_{x_{0}}^{3}(\bar{F})$ for some $k \in \mathbb{N}$ with $\mathcal{P}_{t=1} \not \equiv 0$. Since every non-trivial polynomial has finitely many roots, $\mathcal{P}_{t}$ can only be zero at finitely many values $t \in[0,1]$. By continuity, there is a neighbourhood of $y_{0}$ where this property is satisfied.

\section{Density of Finsler Metrics with Infinite Dimensional Holonomy Group}

In this section, we prove Theorems 1.2 and 1.1.

\subsection{Proof of Theorem 1.2}

We prove the theorem by contradiction: let us suppose that $\mathfrak{g} \subset \mathfrak{X}(U)$ is a finite dimensional Lie algebra on an $n$-dimensional manifold $U$ and it is generating the third order jets at $x_{0} \in U$. As before, the last property means that the 3 -jet projection $\mathfrak{g} \rightarrow J_{x_{0}}^{3}(\mathfrak{X}(U))$ is onto.

We remark that a manifold with a finite dimensional Lie algebra of vector fields with locally transitive action is real analytic (in the sense that there exist a real-analytic atlas such that the vector fields of the Lie algebra are real-analytic). Indeed, a finite dimensional Lie algebra generates a Lie group, and one can provide an analytic atlas on this Lie group so that the group multiplication is analytic. Moreover, local Lie subgroups are real analytic submanifolds, because they are images of the exponential 
map. For more about Lie groups and related topics, we refer to [9,18]. It follows that locally, a manifold with transitive action of a Lie group is the factor group of the Lie group by the stabilizer of one element, which is a local Lie subgroup, then it is also analytic.

We say that the order of singularity of an element $v \in \mathfrak{g}$ at $x_{0}$ is $k \in \mathbb{N}$, noted as $\mathcal{O}_{x_{0}}(v)=k$, if the value and all partial derivatives up to order $k$ at $x_{0}$ are zero, and $v$ has a non-vanishing $(k+1)$ st order derivative. Each nonzero element has finite order by analyticity. Let us consider the set $\mathfrak{g}_{1} \subset \mathfrak{g}$ with order of singularity at least one, that is

$$
\mathfrak{g}_{1}:=\left\{v \in \mathfrak{g} \mid v\left(x_{0}\right)=0, \frac{\partial v}{\partial x_{i}}\left(x_{0}\right)=0, \quad i=1, \ldots, n\right\} .
$$

It is easy to see that $\mathfrak{g}_{1}$ is a Lie subalgebra of $\mathfrak{g}$. Indeed, the $j$ th component of the commutator of two vector fields $v, u \in \mathfrak{g}_{1}$ is given by

$$
[v, u]_{j}=\sum_{i}\left(\frac{\partial v_{j}}{\partial x_{i}} u_{i}-\frac{\partial u_{j}}{\partial x_{i}} v_{i}\right)
$$

and has singularity of order at least two at $x_{0}$. Actually, for any two vector fields $V, U$ from $\mathfrak{g}_{1}$ such that $V$ has order of singularity $k$ and $U$ has order of singularity $m$ their commutator has order of singularity $k+m$.

Since $\mathfrak{g}$ is finite dimensional, so is $\mathfrak{g}_{1}$. It follows that the order of singularity is bounded on $\mathfrak{g}_{1}$. Indeed, if not, then there would be a sequence of vectors in $\mathfrak{g}_{1}$ with strictly monotone increasing order of singularity at $x_{0}$ which would produce an infinite number of linearly independent elements which is impossible.

Let $v_{1} \in \mathfrak{g}_{1}$ be a non-zero element with maximal order $\mathcal{O}_{x_{0}}\left(v_{1}\right)=k$ of singularity at $x_{0}$. Using the 3 -jet generating property, we have $k \geq 2$. Then, for any $v \in \mathfrak{g}_{1}$ we have $\left[v, v_{1}\right] \in \mathfrak{g}_{1}$ and $\mathcal{O}_{x_{0}}\left(\left[v, v_{1}\right]\right)>k$. Since $k$ is maximal, it follows that $\left[v, v_{1}\right]=0$, and it shows that $v_{1}$ commutes with every elements of $\mathfrak{g}_{1}$.

On the other hand, it is possible to choose a point $\hat{x}_{0} \in U$ in a neighbourhood of $x_{0}$ such that $v_{1}\left(\hat{x}_{0}\right) \neq 0$ and $\mathfrak{g}_{1}$ has the 1 -jet generating property at $\hat{x}_{0}$. It follows that one can choose an element $v_{2} \in \mathfrak{g}_{1}$ such that $v_{2}\left(\hat{x}_{0}\right)=0$ but $\mathcal{D}_{v_{1}} v_{2} \neq 0$. Therefore, the commutator $\left[v_{1}, v_{2}\right]$ at $\hat{x}_{0}$ is non-zero. This is a contradiction since $v_{1}$ is an element which commutes with every element of $\mathfrak{g}_{1}$. Theorem 1.2 is proved.

\subsection{Proof of Theorem 1.1}

Let $\mathcal{F}$ be the set of $C^{\infty}$-smooth Finsler metrics on a given manifold $M$ and let us consider the subset $\widetilde{\mathcal{F}} \subset \mathcal{F}$ characterized by the following property: $\tilde{F} \in \widetilde{\mathcal{F}}$ if and only if there exists a point $x_{0} \in M$ such that the curvature vector fields and their derivatives

$$
\left\{\mathcal{R}_{i j}, \nabla_{k} \mathcal{R}_{i j}, \nabla_{k l} \mathcal{R}_{i j}, \nabla_{k l h} \mathcal{R}_{i j} \mid 1 \leq i, j, k, l, h \leq n\right\} \subset \mathfrak{X}\left(\mathcal{I}_{x_{0}}\right),
$$


up to order 3 has the 3 -jet generating property at least at one point of the indicatrix at $x_{0}$. Then

(i) the holonomy group at $x_{0}$ of any $\tilde{F} \in \tilde{\mathcal{F}}$ is infinite dimensional,

(ii) the set $\widetilde{\mathcal{F}}$ is dense in $\mathcal{F}$ with respect to the $C^{m}$ topology for each $m \geq 8$ (in fact for any $m \geq 0$ ),

(iii) the set $\widetilde{\mathcal{F}}$ is open in $\mathcal{F}$ with respect the the $C^{\tilde{m}}$ topology for $\tilde{m} \geq 8$.

Indeed, $(i)$ follows from Theorem 1.2: the infinitesimal holonomy algebra $\mathfrak{h o r}_{x_{0}}^{*}(\tilde{F})$ is infinite dimensional, consequently, the holonomy algebra and the holonomy group of $\tilde{F}$ at $x_{0}$ are infinite-dimensional.

To show (ii), let us consider the Funk-perturbation $F_{t}$ given by (4), a point $x_{0}$ in $M$ and a point $y_{0}$ of the indicatrix at $x_{0}$. By Proposition 4.2 there exists a sufficiently small $t>0$ such that the curvature vector fields and their derivatives up to order 3 has the 3 -jet generating property at $y_{0}$. For sufficiently small $t$ the metric $F_{t}$ is sufficiently close to $F$ in $C^{m}$-topology.

In order to prove (iii), we observe that the jet-generating condition is an open condition, so if it is fulfilled at $y_{0} \in \mathcal{I}_{x_{0}}$, it is fulfilled at any point $y_{1} \in \mathcal{I}_{x_{1}}$ sufficiently close to $y_{0}$ in $C^{m \geq 8}$-topology on $T M$.

Theorem 1.1 is proved.

Remark 5.1 In the proof of Theorem 1.1, it was showed that for a convenient perturbation the infinitesimal holonomy algebra at a point is infinite-dimensional. This remains valid mircrolocally and on the level of germs.

Acknowledgements The cooperation of V.M. with B.H. and Z.M. was started within and was supported by the exchange program of DAAD (PPP Ungarn; Project Number 57447379) and TKA (Project Number 307818), ÚNKP-19-3-I-DE-531 New National Excellence Program of the Ministry for Innovation and Technology. V.M. thanks the University of Debrecen, B.H. and Z.M. thank the Friedrich-Schiller-Universität for their hospitality. We are very grateful to Boris Kruglikov and Peter Olver for useful discussions.

Funding Open access funding provided by University of Debrecen.

Open Access This article is licensed under a Creative Commons Attribution 4.0 International License, which permits use, sharing, adaptation, distribution and reproduction in any medium or format, as long as you give appropriate credit to the original author(s) and the source, provide a link to the Creative Commons licence, and indicate if changes were made. The images or other third party material in this article are included in the article's Creative Commons licence, unless indicated otherwise in a credit line to the material. If material is not included in the article's Creative Commons licence and your intended use is not permitted by statutory regulation or exceeds the permitted use, you will need to obtain permission directly from the copyright holder. To view a copy of this licence, visit http://creativecommons.org/licenses/by/4.0/.

\section{References}

1. Berger, M.: Sur les groupes d'holonomie homogène des variétés à connexion affine et des variétés riemanniennes. Bull. Soc. Math. Fr. 83, 279-330 (1955)

2. Berwald, L.: Untersuchung der Krümmung allgemeiner metrischer Räumen auf Grund des in inher herrschenden Parallelism. Math. Z. 25, 40-73 (1926)

3. Borel, A., Lichnerowicz, A.: Groupes d'holonomie des variétés riemanniennes. C. R. Acad. Sci. Paris 234, 1835-1837 (1952)

4. Chern, S.-S., Shen, Z.: Riemann-Finsler geometry. Nankai Tracts in Mathematics, vol. 426. World Scientific Publishing Co. Pte. Ltd., Hackensack (2005) 
5. Finsler, P.: Ueber Kurven und Flächen in allgemeinen Räumen. Doctoral thesis (1918)

6. Hubicska, B., Muzsnay, Z.: The holonomy groups of projectively flat Randers two-manifolds of constant curvature. Differ. Geom. Appl. 73, 101677 (2020)

7. Hubicska, B., Muzsnay, Z.: Tangent Lie Algebra of a Diffeomorphism Group and Application to Holonomy Theory. J. Geom. Anal. 30, 107-123 (2020)

8. Ivanov, S., Lytchak, A.: Rigidity of Busemann convex Finsler metrics. Comment. Math. Helv. 94, 855-868 (2019)

9. Kolar, I., Michor, P.W., Slovak, J.: Natural Operations in Differential Geometry. Springer-Verlag, Berlin Heidelberg (1993)

10. Kozma, L.: On holonomy groups of Landsberg manifolds. Tensor 62, 87-90 (2000)

11. Matveev, V.S.: On "All regular Landsberg metrics are always Berwald” by Z. I. Szabo. Balkan J. Geom. 14, 50-52 (2009)

12. Matveev, V.S.: Riemannian metrics having common geodesics with Berwald metrics. Publ. Math. Debr. 74, 405-416 (2009)

13. Matveev, V.S., Troyanov, M.: The Binet-Legendre metric in Finsler geometry. Geom. Topol. 16, 2135$2170(2012)$

14. Matveev, V.S.: Geodesically equivalent metrics in general relativity. J. Geom. Phys. 62, 675-691 (2012)

15. Muzsnay, Z., Nagy, P.T.: Projectively flat Finsler manifolds with infinite dimensional holonomy. Forum Math. 27, 767-786 (2015)

16. Muzsnay, Z., Nagy, P.T.: Finsler 2-manifolds with maximal holonomy group of infinite dimension. Diff. Geom. Appl. 39, 1-9 (2015)

17. Olver, P.: Equivalence, Invariants, and Symmetry. Cambridge University Press, Cambridge. ISBN: 0-521-47811-1 (1995)

18. Pontryagin, L.S.: Nepreryvnye Gruppy. Translation: Topological Groups. Gordon and Breach, Philadelphia (1966)

19. Riemann, B.: Über die Hypothesen, welche der Geometrie zu Grunde liegen. Göttingen (1854)

20. Simons, J.: On transitivity of holonomy systems. Ann. Math. 76, 213-234 (1962)

21. Szabó, Z.I.: Positive definite Berwald spaces. Structure theorems on Berwald spaces. Tensor 35(1), 25-39 (1981)

22. Vincze, C.S.: A new proof of Szabó's theorem on the Riemann metrizability of Berwald manifolds. Acta Math. Acad. Paedagog. Nyhazi 21, 199-204 (2005)

Publisher's Note Springer Nature remains neutral with regard to jurisdictional claims in published maps and institutional affiliations. 